\title{
Subharmonic energy-gap structure in superconducting weak links
}

\author{
Flensberg, K.; Hansen, Jørn Bindslev; Octavio, M.
}

\section{Published in:}

Physical Review B

Link to article, DOI:

10.1103/PhysRevB.38.8707

Publication date:

1988

Document Version

Publisher's PDF, also known as Version of record

Link back to DTU Orbit

Citation (APA):

Flensberg, K., Hansen, J. B., \& Octavio, M. (1988). Subharmonic energy-gap structure in superconducting weak links. Physical Review B, 38(13), 8707-8711. https://doi.org/10.1103/PhysRevB.38.8707

\section{General rights}

Copyright and moral rights for the publications made accessible in the public portal are retained by the authors and/or other copyright owners and it is a condition of accessing publications that users recognise and abide by the legal requirements associated with these rights.

- Users may download and print one copy of any publication from the public portal for the purpose of private study or research.

- You may not further distribute the material or use it for any profit-making activity or commercial gain

- You may freely distribute the URL identifying the publication in the public portal

If you believe that this document breaches copyright please contact us providing details, and we will remove access to the work immediately and investigate your claim. 


\title{
Subharmonic energy-gap structure in superconducting weak links
}

\author{
K. Flensberg and J. Bindslev Hansen \\ Physics Laboratory I, The Technical University of Denmark, DK-2800 Lyngby, Denmark \\ M. Octavio \\ Centro de Física, Instituto Venezolano de Investigaciones Científicas, \\ Apartado Postal 21827, Caracas 1020A, Venezuela \\ (Received 2 May 1988)
}

\begin{abstract}
We present corrected calculations of the subharmonic energy-gap structure using the model of Octavio, Tinkham, Blonder, and Klapwijk, which includes the effect of normal scattering in the weak link. We show that while the overall predictions of this model do not change qualitatively, the details of the predicted curves are different and in better agreement with experiment. We also present calculation of the current-voltage characteristics and of the excess currents for $T=0$, as the normal scattering parameter $Z$ is varied. We also show how the calculation can be shortened using symmetry arguments and discuss how this model improves the understanding of the connection between weak links and tunnel junctions.
\end{abstract}

\section{INTRODUCTION}

The subharmonic energy-gap structure (SGS) observed in the current-voltage $(I-V)$ characteristics of superconducting constrictions at voltages $V=2 \Delta / n e$, with $n=1,2, \ldots$ has been a rather difficult problem to understand. Initially explanations of its origin based on multiparticle tunneling ${ }^{1}$ or self-detection of the Josephson radiation, ${ }^{2}$ while predicting correctly the voltage position of the structure, could not reconcile the experimentally observed strengths with theoretical expectations. More recently, Klapwijk, Blonder, and Tinkham ${ }^{3}$ (KBT) showed that the structure could be explained by multiple Andreev reflections at the superconductor-normal-metal $(S-N)$ interfaces at either end of the weak links. This work was extended by Octavio, Tinkham, Blonder, and Klapwijk ${ }^{4}$ (OTBK) who used a Boltzmann-equation approach to remove the difficulties of the earlier trajectory technique and to allow for the inclusion of normal scattering in the superconducting constriction. More recently, Arnold ${ }^{5}$ has calculated the SGS using a modified tunneling Hamiltonian approach which yields results that are at least in qualitative agreement with the OTBK results. Kümmel et al. ${ }^{6}$ have computed the SGS from the Bogoliubov-de Gennes equations, which also include the superconducting channel and should thus yield more complete results than the earlier techniques which did not include information about the superconducting phase. At the same time, comparison with experiment has not been easy due to the wide range of SGS structure that can be observed experimentally depending on whether one studies point contacts or microbridges ${ }^{7,8}$ and on the significant contribution of heating effects to the shape of the curves.

In the course of trying to compare theoretical models to experimental $I-V$ characteristics in niobium point contacts, we found an error in the coefficient for normal reflection in the computer program used by OTBK to calculate their $I-V$ characteristics. While correction of this error does not change the overall qualitative shape of the SGS it does remove some of the detailed discrepancies between theory and experiment and suggests that a more quantitative comparison is indeed possible. We also show
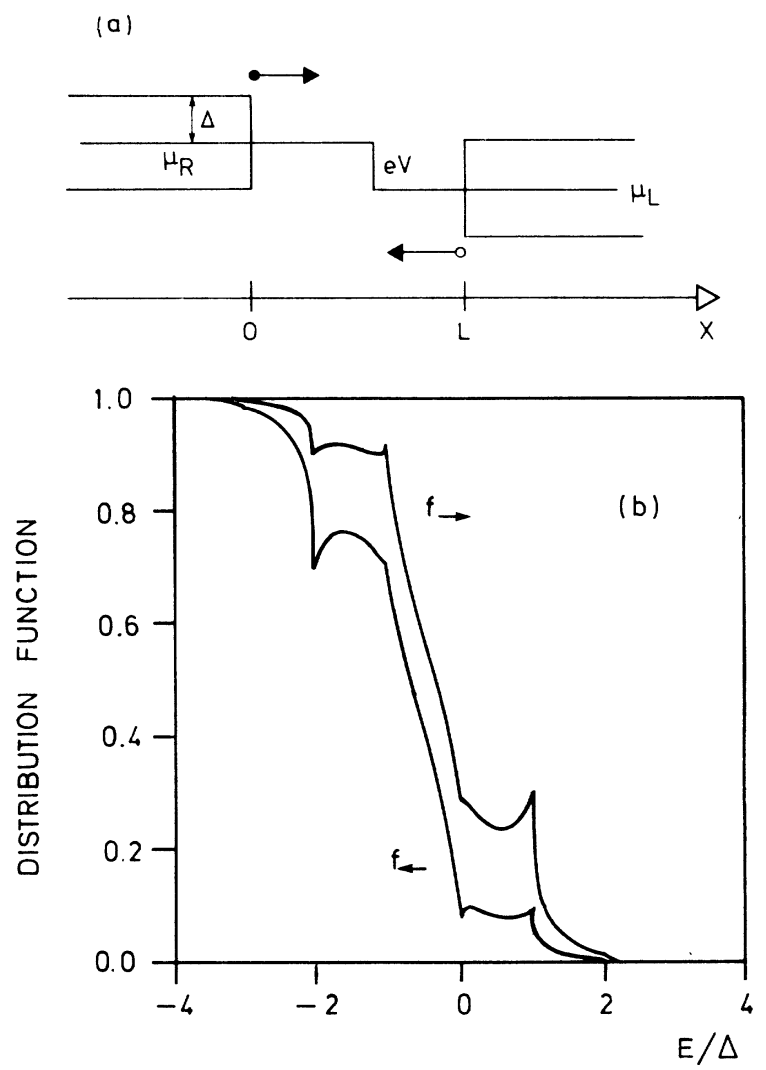

FIG. 1. (a) Schematic diagram of the weak-link model used showing an electron moving towards the right and a hole moving towards the left, $\mu_{R}$ and $\mu_{L}$ are the chemical potentials at either side. (b) Distribution functions $f_{\rightarrow}(E)$ and $f_{\leftarrow}(E)$ for $T / T_{c}=0.70, Z=0.70$, and $e V / \Delta=1$. Note the symmetry in the solution proven in the text. 
how symmetry arguments can be used to simplify the problem and reduce the computation time.

\section{THE OTBK MODEL}

In the OTBK approach the quasiparticles are divided into two subpopulations which depend on their direction of motion $f_{\rightarrow}(E, x)$ and $f_{\leftarrow}(E, x)$, with all energies measured with respect to the local chemical potential. Here $x$ is the spatial variable, with $x=0$ and $L$ defining the ends of the constriction. Applying simple energy arguments to the subpopulations at the opposite end of the constriction in addition to appropriate boundary conditions at the interfaces we have the following equations

$$
\begin{aligned}
f_{\rightarrow}(E, 0)= & A(E)\left[1-f_{\leftarrow}(-E, 0)\right]+B(E) f_{\leftarrow}(E, 0) \\
& +T(E) f_{0}(E), \\
f_{\leftarrow}(E, L)= & A(E)\left[1-f_{\rightarrow}(-E, L)\right]+B(E) f_{\rightarrow}(E, L) \\
& +T(E) f_{0}(E), \\
f_{\rightleftarrows}(E, L)= & f_{\rightleftarrows}(E-e V, 0)
\end{aligned}
$$

$A(E), B(E)$, and $T(E)$ are the coefficients of Andreev reflection, normal reflection and transmission coefficients, respectively. ${ }^{9}$ The second argument in $f(E, x)$ refers to the two ends of an idealized junction, as shown in Fig. 1(a). These three equations can be combined by eliminating one subpopulation to give as in OTBK Eq. (6)

$$
\begin{aligned}
f_{\rightarrow}(E)= & A(E)\left(1-\left\{A(-E+e V)\left[1-f_{\rightarrow}(E-2 e V)\right]+B(-E+e V) f_{\rightarrow}(-E)+T(-E+e V) f_{0}(-E+e V)\right\}\right) \\
& +B(E)\left\{A(E+e V)\left[1-f_{\rightarrow}(-E-2 e V)\right]+B(E+e V) f_{\rightarrow}(E)+T(E+e V) f_{0}(E+e V)\right\}+T(E) f_{0}(E)
\end{aligned}
$$

This equation relates the distribution function $f_{\rightarrow}$ with energy $E$ to those with $(E-2 e V),(-E-2 e V)$, and $-E$ as well as the equilibrium terms $f_{0}(E), f_{0}(E+e V)$, and $f_{0}(-E+e V)$. Since Eq. (4) represents an infinite system, the biggest limitation in its solution is the number of points that can be considered simultaneously, since we need to truncate it while guaranteeing that equilibrium is reached for large and small energies. The solution can nevertheless be simplified further than noted by OTBK by deriving an additional equation from Eqs. (1) and (2). The physical explanation can be given in the following manner: Since the reflection and transmission coefficients are all even in energy, the electrons with distribution $f_{\rightarrow}(E, 0)$ which move to the right in Fig. 1(a) will have exactly the same distribution as the holes of energy $-E$ moving in the opposite direction with distribution $1-f_{\leftarrow}(-E, L)$ and so $f_{\rightarrow}(E, 0)=1-f_{\leftarrow}(-E, L)$ which together with Eq. (3) yields

$$
f_{\rightarrow}(E, 0)=1-f_{\leftarrow}(-E-e V, 0) .
$$

This may be shown formally by considering the boundary conditions. Rewrite Eq. (2) as

$$
\begin{aligned}
1-f_{\leftarrow}(-E-e V)= & A(E) f_{\rightarrow}(E-e V)+B(E) \\
& \times\left[1-f_{\rightarrow}(-E-e V)\right]+T(E) f_{0}(E),
\end{aligned}
$$

where we have used Eq. (3), conservation of probability and the fact that $A(E), B(E)$, and $T(E)$ are all even functions of the energy. It is then easily seen that Eqs. (1) and (6) are equivalent to Eqs. (1) and (5). Now these last two equations are used to eliminate one subpopulation by substitution of Eq. (5) into Eq. (1) to yield

$$
\begin{aligned}
f_{\rightarrow}(E)= & A(E) f_{\rightarrow}(E-e V)+B(E)\left[1-f_{\rightarrow}(-E-e V)\right] \\
& +T(E) f_{0}(E)
\end{aligned}
$$

which needs to be solved self-consistently. Figure 1(b) shows an example of what the distribution functions look like for a particular set of parameters. Equation (7) reduces the computational time due to its much simpler form. One limiting case can be solved, the solution for $B=0(Z=0$ where $Z$ is the dimensionless scattering parameter which models the elastic scattering at the interface $^{6}$ ) is given by

$$
\begin{aligned}
f_{\rightarrow}(E, Z=0)= & {[1-A(E)] f_{0}(E) } \\
& +\sum_{i=1}^{\infty}\left(1-A^{n}\right) f_{0}^{n}(E) \prod_{i=1}^{n} A^{i-1}
\end{aligned}
$$

with the notation $g^{n}(E)=g(E-n e V)$.

The current through the junction is then

$$
\begin{aligned}
I & =\frac{1}{e R_{n}} \int_{-\infty}^{\infty} d E\left[f_{\rightarrow}(E)-f_{\leftarrow}(E)\right] \\
& =\frac{1}{e R_{n}} \int_{-\infty}^{\infty} d E\left[2 f_{\rightarrow}(E)-1\right]
\end{aligned}
$$

where $R_{n}=\left(1+2 Z^{2}\right) R_{0}, \quad$ and $\quad R_{0}=\left[2 \mathcal{A} v_{F} e^{2} N(0)\right]^{-1}$ [here $\mathcal{A}$ is the cross-sectional area of the contact, $v_{F}$ is the Fermi velocity, and $N(0)$ is the density of states at the Fermi energy].

\section{CALCULATIONS}

As noted earlier, an error was present in the normal reflection coefficient in the computer program used by OTBK. Here we present the recalculated derivative 

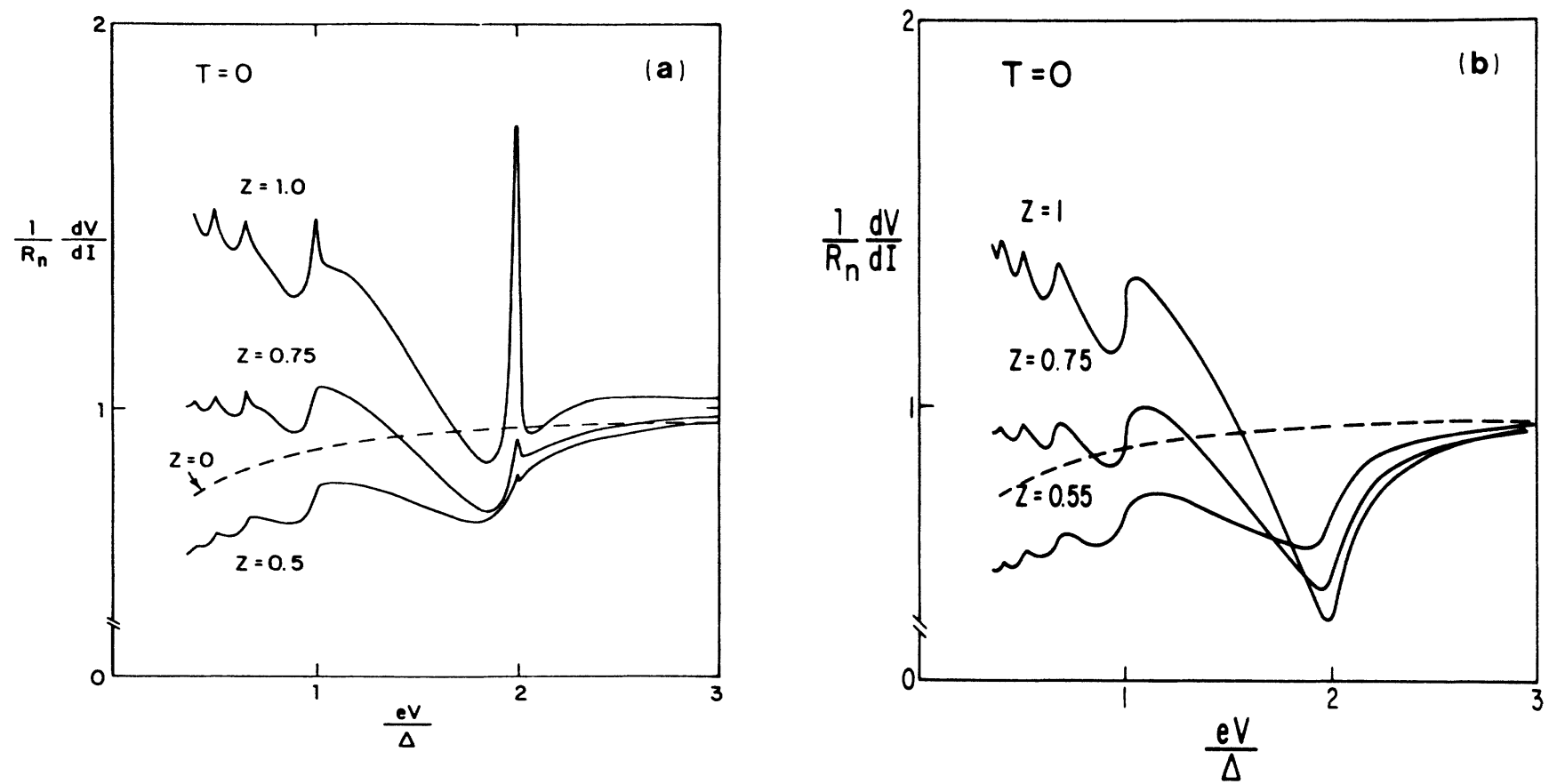

FIG. 2. Normalized differential resistance $\left(1 / R_{n}\right)(d V / d I)$ as a function of the normalized voltage $e V / \Delta$ for $T=0$. (a) Results from OTBK. (b) Corrected results.

curves. $d V / d I$ versus $V$, for the same parameters as OTBK and note the main differences between the results.

Figure 2 shows $\left(1 / R_{n}\right)(d V / d I)$ as a function of the normalized voltage for $T=0$ and varying value of $Z$, parametrizes the degree of normal scattering present in the weak link. In Fig. 2(a) we show the earlier (OTBK)

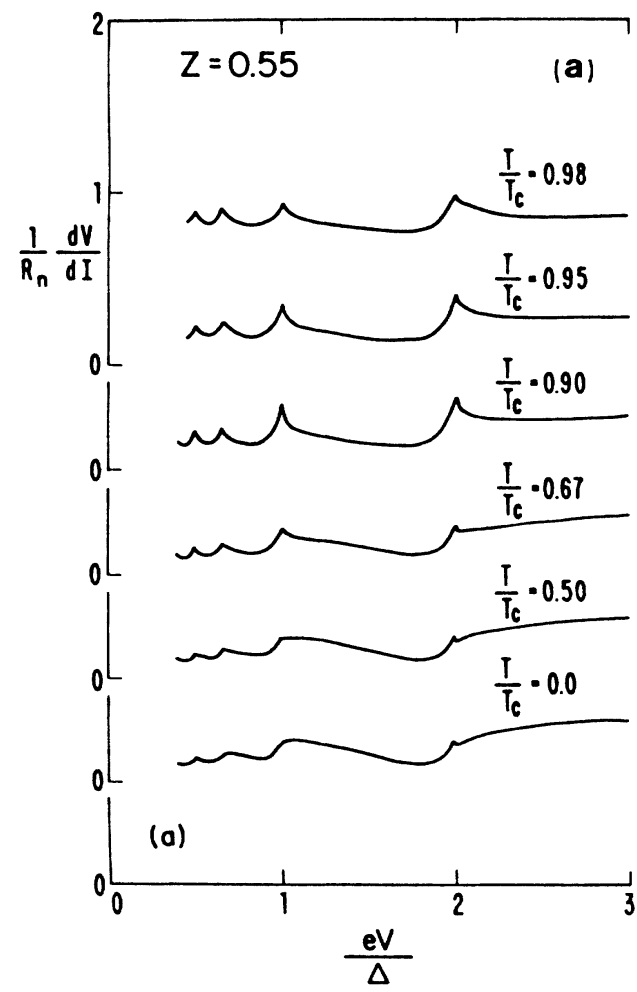

results and in Fig. 2(b) the recalculated curves. As can be seen in the figure the main effect of the correction is to remove the sharp rise in $d V / d I$ at $2 \Delta$ observed earlier as well as some of the sharpness at the maxima in $d V / d I$. In addition, note that the identification of the value of $2 \Delta$ is not clear cut. For low $Z$ values the minimum in $d V / d I$

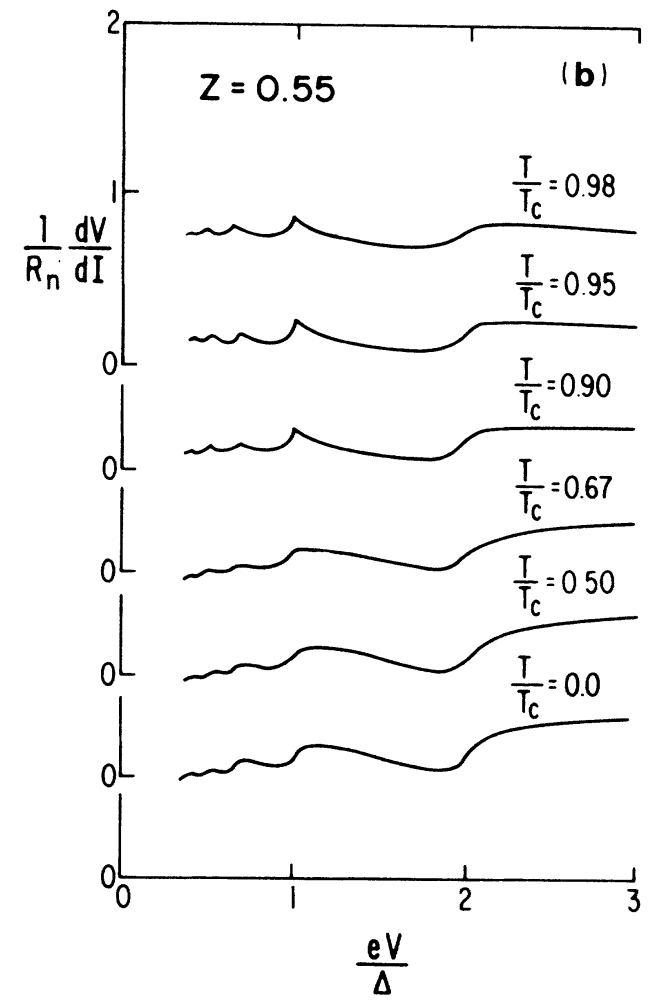

FIG. 3. Normalized differential resistance $\left(1 / R_{n}\right)(d V / d I)$ as a function of the normalized voltage $e V / \Delta$ for $Z=0.55$ and varying temperature (a) OTBK results and (b) corrected results. 

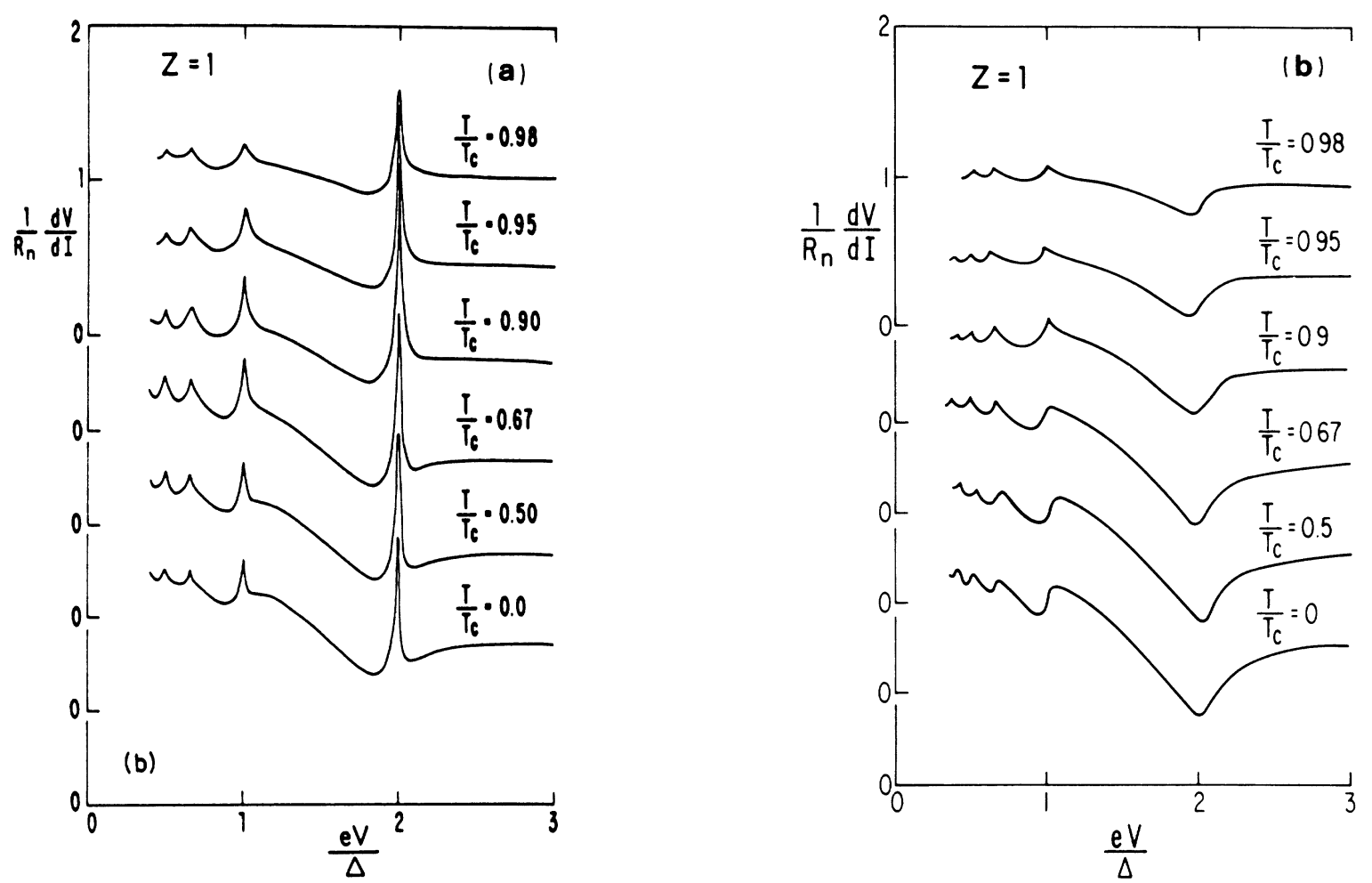

FIG. 4. Normalized differential resistance $\left(1 / R_{n}\right)(d V / d I)$ as a function of the normalized voltage $e V / \Delta$ for $Z=1$ and varying temperature (a) OTBK results. (b) Corrected results.

occurs at a voltage somewhat below $2 \Delta$, this minimum moves closer toward $2 \Delta$ as the value of $Z$ is increased. This effect would give an additional contribution to the heating-induced depression of gap values observed in superconducting microbridges with low $Z$ values. There is a remarkable similarity of our calculated curves with those obtained by Arnold, ${ }^{5}$ including the shift of the minimum with respect to the gap value.

Figure 3 shows $\left(1 / R_{n}\right)(d V / d I)$ as a function of the

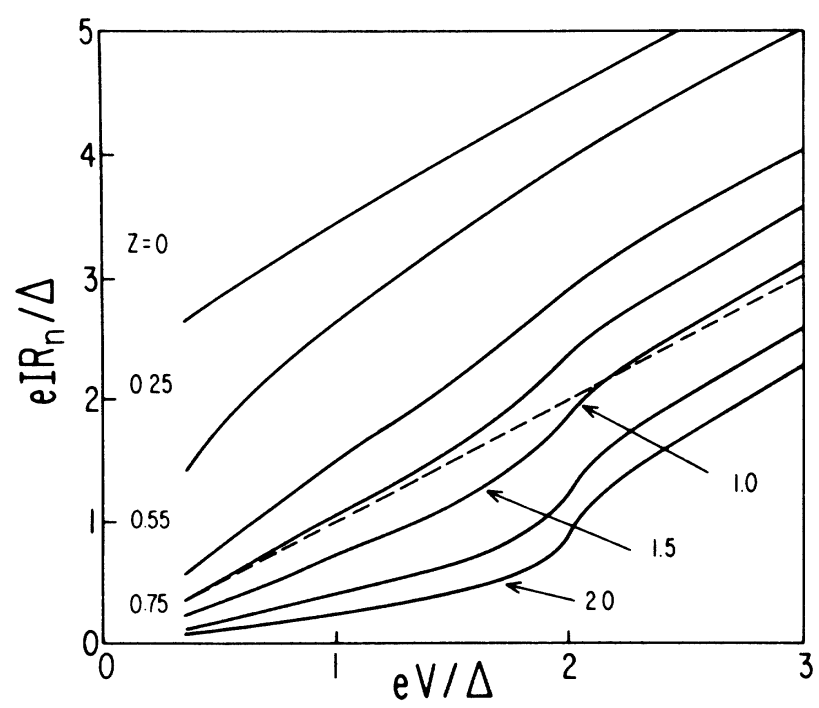

FIG. 5. I-V characteristics as a function of the scattering parameter $Z$, showing the change from excess current to insufficient current as $Z$ is increased. The dashed line corresponds to $V=R_{n} I$. normalized voltage for $Z=0.55$ and varying temperature. Figure 3(a) corresponds to the earlier calculations and Fig. 3(b) to the new calculation. As in Fig. 2 the main effect is to eliminate the sharp peaks and smooth out the curves, but the overall differential resistance remains at the same level. Similar behavior is shown in Fig. 4 for varying temperature and $Z=1$.

Figure 5 shows a series of $I-V$ curves for different

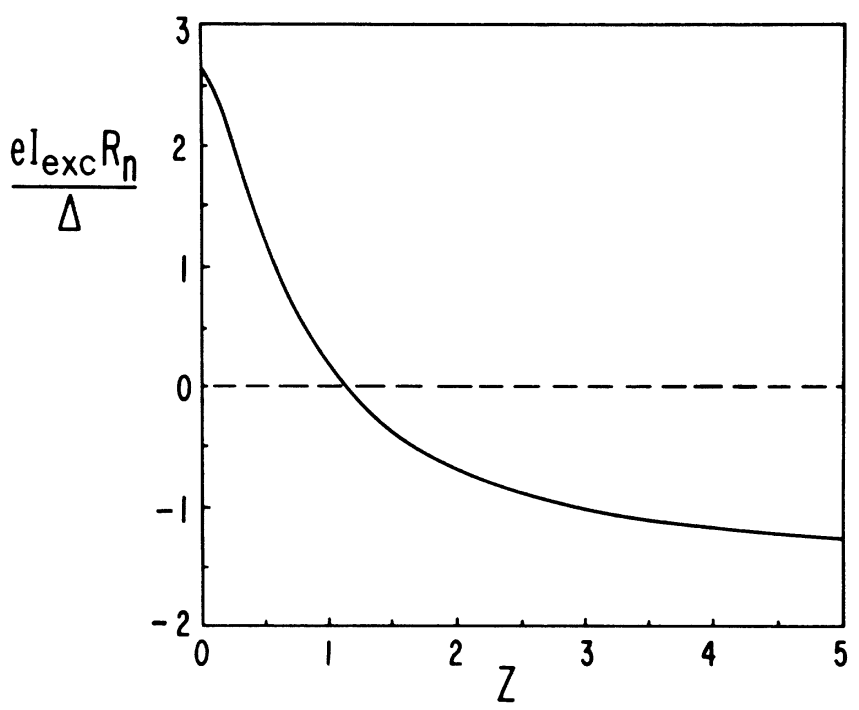

FIG. 6. Plot of the normalized excess current as a function of the scattering parameter $Z$. While the calculation was performed at $T=0$, the temperature dependence is totally contained in the gap function in the normalization and the graph can be used for quick comparison with experiments. 
values of $Z$. For small $Z$ the $I-V$ curves exhibit excess current which means that the high voltage asymptote lies above the normal state characteristic, $V=R_{n} I$. The excess current is defined as $I_{\text {exc }}=I(e V / \Delta \gg>1)-V / R_{n}$. This is in agreement with experimental data for weak links and point contacts and it is the strength of the present model that it can explain both the subharmonic gap structure and the excess current. In Fig. 6 we have made a plot of $I_{\text {exc }}$ versus $Z$. The correction made in this paper reveals another interesting feature; for large $Z$ the excess current becomes a deficient current and thus becomes negative, which can also be seen in Fig. 5 where the curves for large $Z$ lie entirely under the normal state curve. For increasing values of the scattering parameter $Z$, the structure resembles more a "leaky" tunnel junction (e.g., a tunnel junction with localized states in the barrier). Experimentally some tunnel-junction and highresistance point-contact data do in fact exhibit a negative excess current. In the limit where $Z$ goes to infinity the negative excess current converges to $-4 \Delta / 3 e R_{n},{ }^{3}$ which is an analytic result in the limit $\Delta \gg k_{B} T$.

\section{CONCLUSION}

The correction presented in this paper should allow for a more quantitative comparison between experiment and theory. Experimentally, there is a wide range of types of $I-V$ characteristics which can be observed in superconducting weak links. Earlier it seemed difficult to reconcile some of the detailed features of the SGS predicted by our model. These features are no longer present and it should be feasible to compare our results with careful experiments.

In conclusion, we have presented a recalculation of the $I-V$ characteristics of superconducting constrictions using the Boltzmann approach of Octavio, Tinkham, Blonder, and Klapwijk. We have shown how additional arguments can simplify the solution of the problem and how the recalculated curves differ from earlier ones. The recalculated curves are similar to experimental ones and quantitative comparison with experiment should now be possible. ${ }^{10}$ Finally, our results are strikingly like those obtained by Arnold, ${ }^{5}$ using a different approach.

\section{ACKNOWLEDGMENTS}

This work was supported in part by the Consejo $\mathrm{Na}$ cional de Investigaciones Científicas y Tecnológicas (CONICIT), Caracas, Venezuela, under Grant No. S11828 and by the Danish Natural Science Research Council.
1J. R. Schrieffer and J. W. Wilkins, Phys. Rev. Lett. 10, 17 (1963).

${ }^{2}$ N. R. Werthamer, Phys. Rev. 147, 225 (1966).

${ }^{3}$ T. M. Klapwijk, G. E. Blonder, and M. Tinkham, Physica 109-110B + C, 1657 (1982); T. M. Klapwijk, in Proceedings of the Third International Conference on Superconducting Quantum Devices, edited by H. D. Kahlbohm and H. Lübbig (Walter de Gruyter, Berlin, 1985), pp. 2-29.

${ }^{4}$ M. Octavio, M. Tinkham, G. E. Blonder, and T. M. Klapwijk, Phys. Rev. B 27, 6739 (1983).

${ }^{5}$ G. B. Arnold, J.'Low Temp. Phys. 68, 1 (1987).

${ }^{6}$ R. Kümmel, B. Hückestein, and R. Nicolsky, Proceedings of the 18th International Conference on Low Temperature Physics [Jpn. J. Appl. Phys. 26, Suppl. 26-3, 1471 (1987)].

${ }^{7}$ L. J. Barnes, Phys. Rev. 184, 434 (1969); D. A. Weitz, Techni- cal Report No. 14, Division of Applied Sciences, Harvard University, 1978 (unpublished); Yu, Ya. Divin and F. Ya. Nad, Pis'ma Zh. Eksp. Teor. Fiz. 29, 516 (1979); J. Bindslev Hansen, M. Tinkham, and M. Octavio, in Proceedings of the 17th International Conference on Low Temperature Physics, edited by U. Eckern, A. Schmid (North-Holland, Amsterdam, 1984), Pt. I, p. 435.

${ }^{8}$ P. E. Gregers-Hansen, E. Hendricks, M. T. Levinsen, and G. R. Pickett, Phys. Rev. Lett. 31, 524 (1973); M. Octavio, W. J. Skocpol, and M. Tinkham, IEEE Trans. Magn. MAG-1, 739 (1976); I. K. Yanson, Fiz. Nizkikh Temp. 1, 141 (1975) [Sov. J. Low. Temp. Phys. 1, 67 (1975)].

${ }^{9}$ G. E. Blonder, M. Tinkham, and T. M. Klapwijk, Phys. Rev. B 25, 4515 (1982).

10J. Bindslev Hansen and K. Flensberg (unpublished). 